\title{
Vasicek model with mixed-exponential jumps and its applications in finance and insurance
}

Yongfeng $\mathrm{Wu}^{1,2^{*}}$ and Xue Liang ${ }^{3}$

"Correspondence: wyfwyf@126.com 'School of Mathematics and Finance, Chuzhou University, Chuzhou, China

${ }^{2}$ College of Mathematics and Computer Science, Tongling University, Tongling, China Full list of author information is available at the end of the article

\section{空 Springer}

\begin{abstract}
In this paper, the authors study the distribution of the Vasicek model with mixed-exponential jumps and its applications in finance and insurance. With the aid of the piecewise deterministic Markov process theory and the martingale theory, the authors first obtain the explicit forms of the Laplace transforms for the distribution of the Vasicek model with mixed-exponential jumps and its integrated process. As some applications in finance and insurance, the pricing of the default-free zero-coupon bond and the European put option on the zero-coupon bond, and the moments of the aggregate accumulated claim amounts are discussed. The authors also provide some remarks and numerical calculations.
\end{abstract}

MSC: 97K60; 91G40; 91B30

Keywords: Vasicek model with mixed-exponential jumps; Laplace transform; Default-free zero-coupon bond; European put option; Aggregate accumulated claim

\section{Introduction}

Vasicek [1] proposed the following classical Vasicek model which is defined by an equation of the form

$$
\mathrm{d} r_{t}=\alpha\left(\beta-r_{t}\right) \mathrm{d} t+\sigma \mathrm{d} B_{t},
$$

where $\alpha$ is the rate of mean reversion, $\beta$ is the long-run level, $\sigma$ is the volatility coefficient, and $B_{t}$ is a standard Brownian motion.

The Vasicek model is one of the earliest no-arbitrage interest rate models based upon the idea of mean reverting interest rates. It was the first one to capture mean reversion, an essential characteristic of the interest rate that sets it apart from other financial prices. Compared with the CIR model (Cox et al. [2]), the main disadvantage in the Vasicek model is that it is theoretically possible for the interest rate to become negative, an undesirable feature. However, because the explicit solution of the Vasicek model is perfect, some authors still put their attention to the Vasicek model and its generalized versions. Hull and White [3] extended the one-state-variable interest-rate model of Vasicek [1] and showed that the extended Vasicek model is very tractable analytically and is consistent with the current term structure of interest rates or the current volatilities of all forward interest

(c) The Author(s) 2018. This article is distributed under the terms of the Creative Commons Attribution 4.0 International License (http://creativecommons.org/licenses/by/4.0/), which permits unrestricted use, distribution, and reproduction in any medium, provided you give appropriate credit to the original author(s) and the source, provide a link to the Creative Commons license, and indicate if changes were made. 
rates. They compared the option prices obtained using the extended Vasicek model with those obtained in the existing literature. Patie [4] also generalized the Vasicek model and provided its applications in computing the Laplace transform of the price of a European call option. For more research results on the applications of these models in finance and insurance, we can refer the reader to Shimko et al. [5], Mamon [6], Liang et al. [7], Siu [8], Nowman [9], Chen and Hu [10], Qiu et al. [11], Liang et al. [12], Su and Wang [13], Liang et al. [14], Dong [15], Ishitani [16], Xiao et al. [17], Branger et al. [18], Nowak and Romaniuk [19], Chang and Chang [20].

As to the European and American options, Hajipour and Malek [21] proposed a highly accurate method based on non-standard Runge-Kutta (NRK), modified weighted essentially non-oscillatory (MWENO), and grid stretching methods to solve the Black-Scholes equation with discontinuous final condition. The authors believed that this adaptive MWENO method can be extended to solve the American option Black-Scholes equation. Hajipour and Malek [22] presented efficient high-order methods based on weighted essentially non-oscillatory (WENO) technique and backward differentiation formula (BDF) to solve the European and American put options of the Black-Scholes equation. Jajarmi et al. [23] presented the analysis of a hyperchaotic financial system as well as its chaos control and synchronization.

As stated in Beliaeva et al. [24], some scholars, such as Backus et al. [25], Das and Foresi [26], Das [27], Johannes [28], and Piazzesi [29], found that jumps caused by market crashes, interventions by the Federal Reserve, economic surprises, shocks in the foreign exchange markets, and other rare events play a significant role in explaining the dynamics of interest rate changes.

In fact, in practice, there are primary events such as the government' fiscal and monetary policies, the release of corporate financial reports, some natural disasters, terrorist attacks, etc. that will possibly result in some jumps in the interest rate. As time passes, the interest rate process decreases or increases as the firm tries its best to adjust its management after the arrival of a primary event, and so the whole social and economic environment will impel the interest rate to the initial level. This decrease or increase will continue until another event occurs, which will result in another jump in the interest rate process. Therefore, how to describe accurately the above phenomenon of economic activity is an important issue in the interest rate modeling.

In order to describe the appearances of sudden jumps in the interest rate process, we consider the Vasicek model with jumps introduced by Chacko and Das [30], which has the following structure:

$$
\mathrm{d} y_{t}=\alpha\left(\beta-y_{t}\right) \mathrm{d} t+\sigma \mathrm{d} B_{t}+\mathrm{d} J_{t}
$$

where $\alpha, \beta, \sigma$, and $B_{t}$ are as in the previous model (1.1). We assume that $\alpha>0, \beta \geq 0$, and $\sigma \geq 0$. $J_{t}$ is a compound Poisson process which is given by

$$
J_{t}=\sum_{j=1}^{M_{t}} X_{j},
$$

where $M_{t}$ is a Poisson process with frequency $\rho$ and it stands for the total number of jumps up to time $t .\left\{X_{j}, j \geq 1\right\}$ denote the jump sizes and are assumed to be independent and 
identically distributed random variables with distribution function $F(x)$ and probability density function $f_{X}(x)$.

Das [27] showed that the model offers a good statistical description of short rate behavior, and is useful in understanding many empirical phenomena. Some analytical and empirical methods used in this paper support many applications, such as testing for Fed intervention effects, which are shown to be an important source of surprise jumps in interest rates. Beliaeva et al. [24] discussed the pricing of American interest rate options under the Vasicek model with jumps. They provided the pricing of European options on coupon bonds and American options on coupon bonds under this model. Su and Wang [31] investigated the valuation of European option with credit risk in a reduced form model, where the intensity of default is assumed to follow the above extended Vasicek model.

To make our main results of this article more valuable in a practical field, we assume that the jumps sizes in (1.3) follow the mixed-exponential distribution, i.e.,

$$
f_{X}(x)=p_{u} \sum_{i=1}^{m} \lambda_{i} \eta_{i} \mathrm{e}^{-\eta_{i} x} \mathbf{1}_{(x \geq 0)}+q_{d} \sum_{j=1}^{n} q_{j} \theta_{j} \mathrm{e}^{\theta_{j} x} \mathbf{1}_{(x<0)},
$$

where $p_{u} \geq 0, q_{d}=1-p_{u} \geq 0$,

$$
\begin{aligned}
& \lambda_{i} \in(-\infty,+\infty) \quad \text { for all } i=1, \ldots, m, \sum_{i=1}^{m} \lambda_{i}=1, \\
& q_{j} \in(-\infty,+\infty) \quad \text { for all } j=1, \ldots, n, \sum_{j=1}^{n} q_{j}=1, \\
& \eta_{i}>1 \quad \text { for all } i=1, \ldots, m, \text { and } \quad \theta_{j}>0 \text { for all } j=1, \ldots, n .
\end{aligned}
$$

It is clear that the parameters should satisfy some extra conditions to guarantee $f_{X}(x)$ to be a probability density function. As stated in Cai and Kou [32], a necessary condition for $f_{X}(x)$ to be a probability density function is $\lambda_{1}>0, q_{1}>0, \sum_{i=1}^{m} \lambda_{i} \eta_{i} \geq 0$, and $\sum_{j=1}^{n} q_{j} \theta_{j} \geq 0$, and a simple sufficient condition is $\sum_{i=1}^{k} \lambda_{i} \eta_{i} \geq 0$ for all $k=1,2, \ldots, m$ and $\sum_{j=1}^{l} q_{j} \theta_{j} \geq 0$ for all $l=1,2, \ldots, n$.

Cai and Kou [32] pointed out that the mixed-exponential distribution is a very important distribution, which can approximate any distribution in the sense of weak convergence (see Botta and Harris [33]). Actually, even the hyper-exponential jump, which seems only a little narrower than the mixed-exponential jump, cannot be used to approximate all the distribution. Cai and Kou [32] also provided some interesting examples of approximating the gamma distribution, the Pareto distribution, and the Weibull distribution numerically with the mixed-exponential distribution.

It is obvious that (1.1) is a special case of (1.2) for $\rho=0$. In addition, if we take $\beta=\sigma=0$ in model (1.2), it would lead to shot noise process for $y_{t}$. It is well known that the shot noise models have been applied to diverse areas such as finance, insurance, hydrology, and electronics. Therefore, from an applied point of view, it is very significant and nontrivial to study the wider class of Vasicek models with jumps.

Throughout this article, we let $Y_{t}=\int_{0}^{t} y_{u} \mathrm{~d} u$ be the integrated process of $y_{t}$. We also assume that all components dynamics (1.2) and (1.3) are defined on a filtered complete probability space $\{\Omega, \mathcal{F}, Q\}$. In this article, we will first deduce the Laplace transforms of 
the distributions of the processes $y_{t}$ and $Y_{t}$. Then we will present some applications of these Laplace transforms in finance and insurance.

The rest of this article is organized as follows. In Sect. 2, we obtain the Laplace transforms for the Vasicek models with mixed-exponential jumps and their integrated processes. In Sect. 3, by means of the results in the previous section, we discuss the financial application of the Vasicek model with mixed-exponential jumps, and derive the pricing of a default-free zero-coupon bond and a European put option on a zero-coupon bond. In Sect. 4, we investigate the expectation and variance of the aggregate accumulated claim amounts. Some numerical calculations are also given in Sects. 3 and 4. In Sect. 5, some concluding remarks are presented.

\section{The Laplace transforms of the distribution of the Vasicek model with mixed-exponential jumps}

In this section, by means of the piecewise deterministic Markov process theory and the martingale theory, we first deduce the joint Laplace transform of the distribution of the vector process $\left(y_{t}, Y_{t}\right)$, where $y_{t}$ and $Y_{t}$ are defined as in Sect. 1 . Then we obtain the Laplace transforms of the distribution of the Vasicek model with mixed-exponential jumps. As we know, the piecewise deterministic Markov process theory was developed by Davis [34] and has been proved to be a very powerful mathematical tool for examining non-diffusion models. We can refer the reader to find some more details on this theory in Davis [34].

The infinitesimal generator $\mathcal{A}$ of the unique solution to $\operatorname{SDE}$ (1.1) is given by

$$
\mathcal{A} f(y)=\alpha(\beta-y) \frac{\partial f}{\partial y}+\frac{1}{2} \sigma^{2} \frac{\partial^{2} f}{\partial y^{2}}
$$

where $f$ is an arbitrary twice differentiable continuous function. We assume that $y_{t}$ is a Vasicek model with jumps which is a solution of SDE (1.2). By applying the piecewise deterministic Markov process theory and using Theorem 5.5 in Davis [34], one can see that the infinitesimal generator of the process $\left(Y_{t}, y_{t}, t\right)$ acting on a function $f(Y, y, t)$ is given by

$$
\begin{aligned}
\mathcal{A} f(Y, y, t)= & \frac{\partial f}{\partial t}+y \frac{\partial f}{\partial Y}+\alpha(\beta-y) \frac{\partial f}{\partial y}+\frac{1}{2} \sigma^{2} \frac{\partial^{2} f}{\partial y^{2}} \\
& +\rho\left\{\int_{-\infty}^{\infty} f(Y, y+x, t) \mathrm{d} F(x)-f(Y, y, t)\right\},
\end{aligned}
$$

where $f:(0, \infty) \times(0, \infty) \times \mathrm{R}^{+} \rightarrow(0, \infty)$ satisfies:

(1) $f(Y, y, t)$ is bounded on arbitrary finite time intervals;

(2) $f(Y, y, t)$ is differentiable with respect to all $t, y, Y$;

(3)

$$
\left|\int_{-\infty}^{+\infty} f(Y, y+x, t) \mathrm{d} F(x)-f(Y, y, t)\right|<\infty
$$

In order to obtain the joint Laplace transform for the distribution of the vector process $\left(y_{t}, Y_{t}\right)$, we first present a lemma following the method mentioned in Rolski et al. [35]. 
Lemma 2.1 Assume that $m, k$ are two constants such that $k \geq 0$. Then

$$
\exp \left\{-A(t) y_{t}-k Y_{t}+R(t)\right\}
$$

is a martingale where

$$
\begin{aligned}
A(t)= & \left(m-\frac{k}{\alpha}\right) \mathrm{e}^{\alpha t}+\frac{k}{\alpha}, \\
R(t)= & \left(\beta k-\frac{\sigma^{2} k^{2}}{2 \alpha^{2}}\right) t+\left(m-\frac{k}{\alpha}\right)\left(\beta-\frac{k \sigma^{2}}{\alpha^{2}}\right)\left(\mathrm{e}^{\alpha t}-1\right) \\
& -\frac{\sigma^{2}}{4 \alpha}\left(m-\frac{k}{\alpha}\right)^{2}\left(\mathrm{e}^{2 \alpha t}-1\right)+\rho \int_{0}^{t}\{1-h[A(v)]\} \mathrm{d} v, \\
h(\xi)= & \int_{-\infty}^{+\infty} \mathrm{e}^{-\xi x} \mathrm{~d} F(x) .
\end{aligned}
$$

Proof Let

$$
f(Y, y, t)=\exp \{-A(t) y-k Y+R(t)\} .
$$

From Theorem 7.6.1 in Jacobsen [36], $f(Y, y, t)$ has to satisfy $\mathcal{A} f(Y, y, t)=0$ if it is a martingale. Hence, by (2.2), we have

$$
-A^{\prime}(t) y+R^{\prime}(t)-k y-\alpha(\beta-y) A(t)+\frac{1}{2} \sigma^{2} A^{2}(t)+\rho[h(A(t))-1]=0 .
$$

Solving Eq. (2.8), we can get (2.4) and (2.5). The proof is completed.

Now, by means of Lemma 2.1, we give the joint Laplace transform of the distribution of the vector process $\left(y_{t}, Y_{t}\right)$.

Theorem 2.1 Assume that $\mu, k$ are two constants and that $f_{X}(x)$ satisfies (1.4). Then the joint Laplace transform of the distribution of $\left(y_{t}, Y_{t}\right)$ is given by

$$
E^{Q}\left\{\mathrm{e}^{-\mu y_{t}} \mathrm{e}^{-k\left(Y_{t}-Y_{s}\right)} \mid y_{s}\right\}=\exp \left\{-B(s, t ; \mu, k) y_{s}+C(s, t ; \mu, k)\right\},
$$

where

$$
\begin{aligned}
B(s, t ; \mu, k)= & \left(\mu-\frac{k}{\alpha}\right) \mathrm{e}^{\alpha(s-t)}+\frac{k}{\alpha}, \\
C(s, t ; \mu, k)= & \left(\rho+\beta k-\frac{\sigma^{2} k^{2}}{2 \alpha^{2}}\right)(s-t) \\
& +\left(\beta-\frac{k \sigma^{2}}{\alpha^{2}}\right)[B(s, t ; \mu, k)-\mu]-\frac{\sigma^{2}}{4 \alpha}\left(\mu-\frac{k}{\alpha}\right)^{2}\left[\mathrm{e}^{2 \alpha(s-t)}-1\right] \\
& +\rho p_{u} \sum_{i=1}^{m} \frac{\lambda_{i} \eta_{i}}{k+\alpha \eta_{i}} \ln \left[\frac{\left(k+\alpha \eta_{i}\right) \mathrm{e}^{\alpha(t-s)}+(\alpha \mu-k)}{\alpha\left(\mu+\eta_{i}\right)}\right] \\
& -\rho q_{d} \sum_{j=1}^{n} \frac{q_{j} \theta_{j}}{k-\alpha \theta_{j}} \ln \left[\frac{\left(k-\alpha \theta_{j}\right) \mathrm{e}^{\alpha(t-s)}+(\alpha \mu-k)}{\alpha\left(\mu-\theta_{j}\right)}\right] .
\end{aligned}
$$


Proof By Lemma 2.1, for an arbitrary fixed time $t^{*}\left(0 \leq s \leq t^{*}\right)$, we have

$$
E^{Q}\left\{\exp \left\{-A\left(t^{*}\right) y_{t^{*}}-k Y_{t^{*}}+R\left(t^{*}\right)\right\} \mid y_{s}\right\}=\exp \left\{-A(s) y_{s}-k Y_{s}+R(s)\right\} .
$$

Then we get

$$
E^{Q}\left\{\exp \left\{-A\left(t^{*}\right) y_{t^{*}}-k\left(Y_{t^{*}}-Y_{s}\right)\right\} \mid y_{s}\right\}=\exp \left\{-A(s) y_{s}+R(s)-R\left(t^{*}\right)\right\} .
$$

Set $A\left(t^{*}\right)=\mu \geq 0$. By (2.4), we get

$$
m=\left(\mu-\frac{k}{\alpha}\right) \mathrm{e}^{\alpha t^{*}}+\frac{k}{\alpha}
$$

Then we can get

$$
\begin{aligned}
& A(s)=\left(\mu-\frac{k}{\alpha}\right) \mathrm{e}^{\alpha\left(s-t^{*}\right)}+\frac{k}{\alpha}=B\left(s, t^{*} ; \mu, k\right), \\
& A(v)=\left(\mu-\frac{k}{\alpha}\right) \mathrm{e}^{\alpha\left(v-t^{*}\right)}+\frac{k}{\alpha}=B\left(v, t^{*} ; \mu, k\right)
\end{aligned}
$$

and

$$
\begin{aligned}
R(s)-R\left(t^{*}\right)= & \left(\rho+\beta k-\frac{\sigma^{2} k^{2}}{2 \alpha^{2}}\right)\left(s-t^{*}\right)+\left(\beta-\frac{k \sigma^{2}}{\alpha^{2}}\right)\left[B\left(s, t^{*} ; \mu, k\right)-\mu\right] \\
& -\frac{\sigma^{2}}{4 \alpha}\left(\mu-\frac{k}{\alpha}\right)^{2}\left[\mathrm{e}^{2 \alpha\left(s-t^{*}\right)}-1\right]+\rho \int_{s}^{t^{*}} h\left[B\left(\nu, t^{*} ; \mu, k\right)\right] \mathrm{d} v .
\end{aligned}
$$

Let $u=t^{*}-v$ in (2.15), we have

$$
\rho \int_{s}^{t^{*}} h\left[B\left(v, t^{*} ; \mu, k\right)\right] \mathrm{d} v=\rho \int_{0}^{t^{*}-s} h[B(0, u ; \mu, k)] \mathrm{d} u .
$$

From $A\left(t^{*}\right)=\mu,(2.12)-(2.16)$, we have

$$
\begin{aligned}
E^{Q}\left\{\mathrm{e}^{-\mu y_{t^{*}}} \mathrm{e}^{-k\left(Y_{\left.t^{*}-Y_{s}\right)}\right.} \mid y_{s}\right\} \\
=\exp \left\{-B\left(s, t^{*} ; \mu, k\right) y_{s}+\left(\rho+\beta k-\frac{\sigma^{2} k^{2}}{2 \alpha^{2}}\right)\left(s-t^{*}\right)+\left(\beta-\frac{k \sigma^{2}}{\alpha^{2}}\right)\left[B\left(s, t^{*} ; \mu, k\right)-\mu\right]\right. \\
\left.\quad-\frac{\sigma^{2}}{4 \alpha}\left(\mu-\frac{k}{\alpha}\right)^{2}\left[\mathrm{e}^{2 \alpha\left(s-t^{*}\right)}-1\right]+\rho \int_{0}^{t^{*}-s} h[B(0, u ; \mu, k)] \mathrm{d} u\right\} .
\end{aligned}
$$

By some standard computations, we have

$$
\begin{aligned}
h[B(0, u ; \mu, k)]= & p_{u} \sum_{i=1}^{m} \frac{\lambda_{i} \eta_{i} \mathrm{e}^{\alpha u}}{\left(k / \alpha+\eta_{i}\right) \mathrm{e}^{\alpha u}+(\mu-k / \alpha)} \\
& -q_{d} \sum_{j=1}^{n} \frac{q_{j} \theta_{j} \mathrm{e}^{\alpha u}}{\left(k / \alpha-\theta_{j}\right) \mathrm{e}^{\alpha u}+(\mu-k / \alpha)} .
\end{aligned}
$$


Hence

$$
\begin{aligned}
\rho \int_{0}^{t^{*}-s} h[B(0, u ; \mu, k)] \mathrm{d} u= & \rho p_{u} \sum_{i=1}^{m} \frac{\lambda_{i} \eta_{i}}{k+\alpha \eta_{i}} \ln \left[\frac{\left(k+\alpha \eta_{i}\right) \mathrm{e}^{\alpha\left(t^{*}-s\right)}+(\alpha \mu-k)}{\alpha\left(\mu+\eta_{i}\right)}\right] \\
& -\rho q_{d} \sum_{j=1}^{n} \frac{q_{j} \theta_{j}}{k-\alpha \theta_{j}} \ln \left[\frac{\left(k-\alpha \theta_{j}\right) \mathrm{e}^{\alpha\left(t^{*}-s\right)}+(\alpha \mu-k)}{\alpha\left(\mu-\theta_{j}\right)}\right] .
\end{aligned}
$$

Plugging (2.18) into the integral term of (2.17), we get

$$
\begin{aligned}
E^{Q}\left\{\mathrm{e}^{-\mu y_{t^{*}}} \mathrm{e}^{-k\left(Y_{\left.t^{*}-Y_{s}\right)}\right)} \mid y_{s}\right\} & =\exp \left\{-B\left(s, t^{*} ; \mu, k\right) y_{s}+\left(\rho+\beta k-\frac{\sigma^{2} k^{2}}{2 \alpha^{2}}\right)\left(s-t^{*}\right)\right. \\
& +\left(\beta-\frac{k \sigma^{2}}{\alpha^{2}}\right)\left[B\left(s, t^{*} ; \mu, k\right)-\mu\right]-\frac{\sigma^{2}}{4 \alpha}\left(\mu-\frac{k}{\alpha}\right)^{2}\left[\mathrm{e}^{2 \alpha\left(s-t^{*}\right)}-1\right] \\
& +\rho p_{u} \sum_{i=1}^{m} \frac{\lambda_{i} \eta_{i}}{k+\alpha \eta_{i}} \ln \left[\frac{\left(k+\alpha \eta_{i}\right) \mathrm{e}^{\alpha\left(t^{*}-s\right)}+(\alpha \mu-k)}{\alpha\left(\mu+\eta_{i}\right)}\right] \\
& \left.-\rho q_{d} \sum_{j=1}^{n} \frac{q_{j} \theta_{j}}{k-\alpha \theta_{j}} \ln \left[\frac{\left(k-\alpha \theta_{j}\right) \mathrm{e}^{\alpha\left(t^{*}-s\right)}+(\alpha \mu-k)}{\alpha\left(\mu-\theta_{j}\right)}\right]\right\} .
\end{aligned}
$$

Since $t^{*}$ is arbitrary, (2.19) remains true for all $t \geq s \geq 0$, which implies (2.9). The proof is completed.

Setting $k=0$ and $\mu=0$ in (2.9), respectively, we obtain the following corollary.

Corollary 2.1 Assume that $\mu, k$ are two constants and that $f_{X}(x)$ satisfies (1.4). Then the Laplace transforms of the distributions of $y_{t}$ and $Y_{t}$ are respectively given by

$$
E^{Q}\left\{\mathrm{e}^{-\mu y_{t}} \mid y_{s}\right\}=\exp \left\{-B(s, t ; \mu, 0) y_{s}+C(s, t ; \mu, 0)\right\}
$$

and

$$
E^{Q}\left\{\mathrm{e}^{-k\left(Y_{t}-Y_{s}\right)} \mid y_{s}\right\}=\exp \left\{-B(s, t ; 0, k) y_{s}+C(s, t ; 0, k)\right\}
$$

Remark2.1 If we take $s=\beta=\sigma=0, p_{u}=1, q_{d}=0$, and $m=2$ in (2.20), we can get directly

$$
E^{Q}\left\{\mathrm{e}^{-\mu y_{t}} \mid y_{0}\right\}=\exp \left\{-\mu \mathrm{e}^{-\alpha t} y_{0}\right\}\left(\frac{\eta_{1}+\mu \mathrm{e}^{-\alpha t}}{\eta_{1}+\mu}\right)^{\frac{\rho \lambda_{1}}{\alpha}}\left(\frac{\eta_{2}+\mu \mathrm{e}^{-\alpha t}}{\eta_{2}+\mu}\right)^{\frac{\rho \lambda_{2}}{\alpha}},
$$

which is actually the conclusion (9) of Jang [37].

\section{Applications in finance}

In this section, we state some financial applications of the Vasicek model with mixedexponential jumps (1.2). Based on the results obtained in the previous section, we derive the pricing of a default-free zero-coupon bond and a European put option on a zerocoupon bond. We also present some numerical calculations in the section. 


\subsection{Pricing a default-free zero-coupon bond}

We first present the bond pricing formula.

Proposition 3.1 Assume that the interest rate $r_{t}$ satisfies dynamics (1.2), (1.3), and (1.4). Then the pricing of the discount zero-coupon bond at time $t(0<t<T)$ is given by

$$
P(t, T)=\exp \left\{-B(t, T ; 0,1) r_{t}+C(t, T ; 0,1)\right\},
$$

where $T$ is the expiration date of the zero-coupon bond.

Proof Note that $P(t, T)=E^{Q}\left\{\mathrm{e}^{-\int_{t}^{T} r_{s} \mathrm{~d} s} \mid r_{s}\right\}$. Then (3.1) follows immediately from Corollary 2.1. The proof is completed.

Without loss of generality, we consider a zero-coupon bond paying 1 at time $T$. The present value of the default-free zero-coupon bond at time 0 paying 1 at time $T$ is given by

$$
P(0, T)=E^{Q}\left\{\mathrm{e}^{-\int_{0}^{T} r_{s} \mathrm{~d} s} \mid r_{0}\right\}
$$

Applying Proposition 3.1, we give the following example to illustrate the calculation of the price of the default-free zero-coupon bond.

Example 3.1 The parameter values in (3.1) used to calculate the price of the default-free zero-coupon bond are

$$
\begin{aligned}
& t=0, \quad T=1, \quad \eta_{1}=2, \quad \eta_{2}=1.5, \quad \theta_{1}=2, \quad \theta_{2}=1.5, \\
& m=n=2, \quad p_{u}=0.6, \quad q_{d}=0.4, \quad \lambda_{1}=1.2, \quad \lambda_{2}=-0.2, \\
& q_{1}=1.3, \quad q_{2}=-0.3, \quad \alpha=2, \quad \beta=0.5, \quad r_{0}=0.03, \quad \rho=3, \quad \sigma=2 .
\end{aligned}
$$

Then the default-free zero-coupon bond price is given by

$$
P(0,1)=E^{Q}\left\{\mathrm{e}^{-\int_{0}^{1} r_{s} \mathrm{~d} s} \mid r_{0}\right\}=0.8662 .
$$

If we use a deterministic interest rate $r=0.05$, the default-free zero-coupon bond price is

$$
\mathrm{e}^{-0.05}=0.9512
$$

In order to illuminate the dynamic relationship between $P(0,1)$ and the parameters $\rho$, $\sigma, \eta_{1}, \eta_{2}, \theta_{1}$, and $\theta_{2}$, we give Fig. 1 .

Remark 3.1 In this paper we can find that the higher the frequency $\rho$ is, the lower the price of the default-free zero-coupon bond is (see Fig. 1(a)). From Fig. 1(b), we also can find that the higher the initial interest rate is, the lower the price of the default-free zero-coupon bond is. Similarly, the bigger the magnitude of the positive jump is, the less attractive purchasing a default-free zero-coupon bond is. Thus the smaller $\eta_{1}$ and $\eta_{2}$ are, the lower the price of the default-free zero-coupon bond is (see Fig. 1(c)). In addition, the higher volatility coefficient $\sigma$ is, the higher the price of the default-free zero-coupon bond is (see Fig. 1(d)). 


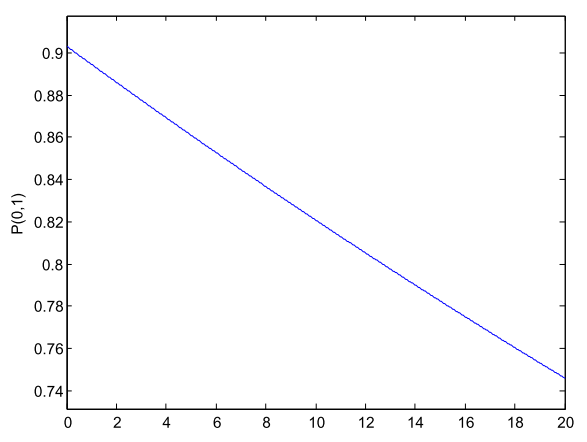

(a) relationship between $P(0,1)$ and $\rho$

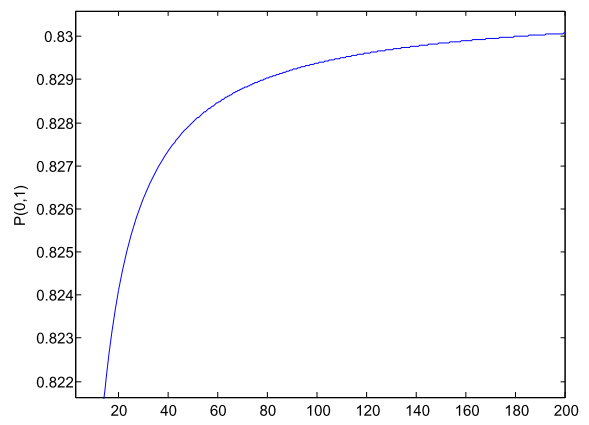

(c) relationship between $P(0,1)$ and $\eta_{i}$ $\theta_{i}, \mathrm{i}=1,2$

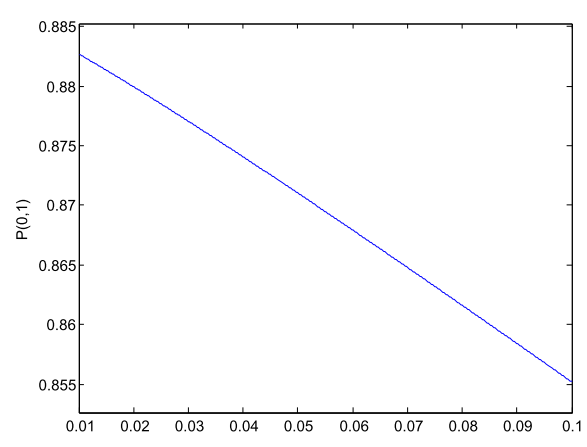

(b) relationship between $P(0,1)$ and $r_{0}$

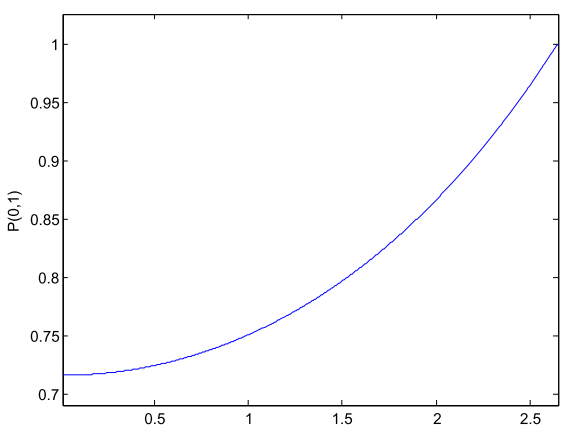

(d) relationship between $P(0,1)$ and $\sigma$

Figure 1 The dynamic relationships between $P(0,1)$ and the other parameters

\subsection{Pricing a European put option on a zero-coupon bond}

We assume that the interest rate dynamics are described as in (1.2), (1.3), and (1.4). Now we investigate the pricing of a European put option on a zero-coupon bond.

As we know, $\max \left\{K-P\left(T_{0}, T\right), 0\right\}$ is the payoff at the expiration date $T_{0}$ of a European put option on a zero-coupon bond. Here, $K$ is the strike price of the European put option. Then the price at time 0 of the European put option on the zero-coupon bond is

$$
P_{0}=E^{Q}\left[\mathrm{e}^{-\int_{0}^{T_{0}} r_{s} \mathrm{~d} s} \max \left\{K-P\left(T_{0}, T\right), 0\right\}\right] .
$$

Proposition 3.2 Assume that the interest rate $r_{t}$ satisfies dynamics (1.2), (1.3), and (1.4). Then the pricing of the European put option on the zero-coupon bond is given by

$$
P_{0}=K P\left(0, T_{0}\right) \Psi_{1}-P(0, T) \Psi_{2},
$$

where

$$
\begin{aligned}
\Psi_{i}= & \frac{1}{2}+\frac{1}{\pi} \int_{0}^{+\infty} \Re\left(\frac{\mathrm{e}^{-i u r_{*}} \phi_{i}(u)}{i u}\right) \mathrm{d} u, \quad i=1,2, \\
r_{*}= & \frac{C\left(T_{0}, T ; 0,1\right)-\ln K}{B\left(T_{0}, T ; 0,1\right)}, \\
\phi_{1}(u)= & \exp \left\{C\left(0, T_{0} ; i u, 1\right)-C\left(0, T_{0} ; 0,1\right)+\left(B\left(0, T_{0} ; 0,1\right)-B\left(0, T_{0} ; i u, 1\right)\right) r_{0}\right\}, \\
\phi_{2}(u)= & \exp \left\{C\left(0, T_{0} ; i u-B\left(T_{0}, T ; 0,1\right), 1\right)+C\left(T_{0}, T ; 0,1\right)\right. \\
& \left.\quad-C(0, T ; 0,1)+\left(B(0, T ; 0,1)-B\left(0, T_{0} ; i u-B\left(T_{0}, T ; 0,1\right), 1\right)\right) r_{0}\right\} .
\end{aligned}
$$


Proof It is clear that the expectation in (3.2) can be divided into the following two expressions:

$$
\begin{aligned}
P_{0}= & K E^{Q}\left[\mathrm{e}^{-\int_{0}^{T_{0}} r_{s} \mathrm{~d} s} \mathbf{1}_{\left(P\left(T_{0}, T\right) \leq K\right)}\right] \\
& -E^{Q}\left[\mathrm{e}^{-\int_{0}^{T_{0}} r_{s} \mathrm{~d} s} P\left(T_{0}, T\right) \mathbf{1}_{\left(P\left(T_{0}, T\right) \leq K\right)}\right] \\
= & K P\left(0, T_{0}\right) E^{Q}\left[\frac{\mathrm{e}^{-\int_{0}^{T_{0}} r_{s} \mathrm{~d} s}}{P\left(0, T_{0}\right)} \mathbf{1}_{\left(r_{T_{0}} \geq r_{*}\right)}\right] \\
& -P(0, T) E^{Q}\left[\frac{\mathrm{e}^{-\int_{0}^{T_{0}} r_{s} \mathrm{~d} s} P\left(T_{0}, T\right)}{P(0, T)} \mathbf{1}_{\left(r_{T_{0}} \geq r_{*}\right)}\right] .
\end{aligned}
$$

By means of the methods used in Duffie et al. [38], we can accomplish the calculations of the above expectations. We consider two new measures $Q_{1}$ and $Q_{2}$ which are all equivalent to measure $Q$. The Radon-Nikodym derivatives are defined as

$$
\frac{\mathrm{d} Q_{1}}{\mathrm{~d} Q}=\frac{\mathrm{e}^{-\int_{0}^{T_{0}} r_{s} \mathrm{~d} s}}{P\left(0, T_{0}\right)} \quad \text { and } \quad \frac{\mathrm{d} Q_{2}}{\mathrm{~d} Q}=\frac{\mathrm{e}^{-\int_{0}^{T_{0}} r_{s} \mathrm{~d} s} P\left(T_{0}, T\right)}{P(0, T)} .
$$

Then, under the new measures $Q_{1}$ and $Q_{2}$, we have

$$
P_{0}=K P\left(0, T_{0}\right) Q_{1}\left(r_{T_{0}} \geq r_{*}\right)-P(0, T) Q_{2}\left(r_{T_{0}} \geq r_{*}\right)=K P\left(0, T_{0}\right) \Psi_{1}-P(0, T) \Psi_{2},
$$

where

$$
\Psi_{i}=Q_{i}\left(r_{T_{0}} \geq r_{*}\right)=\frac{1}{2}+\frac{1}{\pi} \int_{0}^{+\infty} \Re\left(\frac{\mathrm{e}^{-i u r_{*}} \phi_{i}(u)}{i u}\right) \mathrm{d} u, \quad i=1,2,
$$

which can be obtained by following the methods of Duffie et al. [38]. More details can be found in Appendices in Duffie et al. [38].

Under the measures $Q_{1}$ and $Q_{2}$, the characteristic functions of random variable $r_{T_{0}}$ are defined as $\phi_{1}(u)$ and $\phi_{2}(u)$, which are given by

$$
\phi_{1}(u)=E^{Q_{1}}\left[\mathrm{e}^{i u r_{0}}\right]=E^{Q}\left[\frac{\mathrm{e}^{-\int_{0}^{T_{0}} r_{s} \mathrm{~d} s}}{P\left(0, T_{0}\right)} \mathrm{e}^{i u r_{T_{0}}}\right]=\frac{1}{P\left(0, T_{0}\right)} E^{\mathrm{Q}}\left[\mathrm{e}^{-\int_{0}^{T_{0}} r_{s} \mathrm{~d} s+i u r_{T_{0}}}\right]
$$

and

$$
\begin{aligned}
\phi_{2}(u) & =E^{Q_{2}}\left[\mathrm{e}^{i u r_{T_{0}}}\right]=E^{Q}\left[\frac{\mathrm{e}^{-\int_{0}^{T_{0}} r_{s} \mathrm{ds}} P\left(T_{0}, T\right)}{P(0, T)} \mathrm{e}^{i u r_{T_{0}}}\right] \\
& =\frac{\exp \left\{C\left(T_{0}, T ; 0,1\right)\right\}}{P(0, T)} E^{Q}\left[\mathrm{e}^{-\int_{0}^{T_{0}} r_{s} \mathrm{~d} s+\left(i u-B\left(T_{0}, T ; 0,1\right)\right) r_{T_{0}}}\right] .
\end{aligned}
$$

Therefore, from Proposition 3.1 and Theorem 2.1, (3.6) and (3.7) can be obtained directly. Here we omit the details. The proof is completed.

Then we present the following example to illustrate the calculation of the price of the European put option on the zero-coupon bond. We consider a one-year European put 
Table 1 European put option prices and varying parameter values

\begin{tabular}{lllllll}
\hline Parameters & $\sigma=0.2$ & $\sigma=0.4$ & $\rho=40$ & $\rho=60$ & $r_{0}=0.04$ & $r_{0}=0.06$ \\
\hline$P_{0}$ & 0.2097 & 0.1763 & 0.2043 & 0.2146 & 0.2198 & 0.2180 \\
$P_{0}^{\prime}$ & 0.0260 & 0.0249 & 0.0256 & 0.0256 & 0.0257 & 0.0254 \\
Parameters & $\eta_{1}=\theta_{1}=3$ & $\eta_{1}=\theta_{1}=30$ & $\beta=0.08$ & $\beta=0.10$ & $K=0.2$ & $K=0.4$ \\
& $\eta_{2}=\theta_{2}=2$ & $\eta_{2}=\theta_{2}=20$ & & & & \\
$P_{0}$ & 0.1231 & 0.0816 & 0.2202 & 0.2166 & 0.1477 & 0.1316 \\
$P_{0}^{\prime}$ & 0.0257 & 0.0257 & 0.0236 & 0.0276 & 0.0257 & 0.0257 \\
\hline
\end{tabular}

option on a five-year zero-coupon bond. The zero-coupon bond pays one unit of currency to the buyer of the bond at maturity time.

Example 3.2 The parameter values in (3.3) are given by

$$
\begin{array}{lcccc}
m=n=2, & p_{u}=0.6, \quad q_{d}=0.4, \quad \alpha=2, \quad \beta=0.09, \quad \sigma=0.3, \\
\rho=50, & \eta_{1}=\theta_{1}=15, \quad \eta_{2}=\theta_{2}=10, \quad r_{0}=0.05, \quad K=0.3, & \\
\lambda_{1}=1.2, \quad \lambda_{2}=-0.2, \quad q_{1}=1.3, \quad q_{2}=-0.3, \quad T=5, \quad T_{0}=1 .
\end{array}
$$

In order to illuminate the dynamic relationships between the price of the European put option on the zero-coupon bond and some varying parameter values in this model, we present Table 1.

In Table $1, P_{0}^{\prime}$ denotes the price of a European put option on the zero-coupon bond in the pure diffusion model (1.1).

Remark 3.2 In Example 3.2, we discuss the prices of the European put option on the zerocoupon bond with some varying parameter values in model (1.2). Table 1 indicates the following facts. Firstly, because both the volatility from the diffusion term $\sigma \mathrm{d} B_{t}$ and that from the jump term $\mathrm{d} J_{t}$ have a positive effect on the option prices, the prices in the jumpdiffusion model are higher than those in the pure diffusion model. Secondly, the varying values of the volatility coefficient have an apparent effect on the option prices. As shown in Table 1, the higher volatility coefficient will cause higher prices of the underlying asset (the zero-coupon bond). Thus the higher volatility coefficient $\sigma$ is, the lower the option price is. Finally, we can find that higher frequency $\rho$ in the Poisson process leads to higher option prices. The reason is that higher frequency will result in lower prices of the zerocoupon bond.

\section{Application in insurance}

In this section, we discuss the applications of model (1.2) in insurance. By means of the conclusions of Sect. 2, we will deduce the expectation and variance of the process $y_{t}$.

Proposition 4.1 Assume that the process $y_{t}$ satisfies dynamics (1.2), (1.3), and (1.4). Then the expectation of $y_{t}$ is given by

$$
E^{Q}\left(y_{t} \mid y_{0}\right)=\mathrm{e}^{-\alpha t} y_{0}-\left[\beta+\frac{\rho}{\alpha}\left(p_{u} \sum_{i=1}^{m} \frac{\lambda_{i}}{\eta_{i}}+q_{d} \sum_{j=1}^{n} \frac{q_{j}}{\theta_{j}}\right)\right]\left(\mathrm{e}^{-\alpha t}-1\right) .
$$


Proof Let $s=0$ in (2.20), we have

$$
\begin{aligned}
E^{Q}\left\{\mathrm{e}^{-\mu y_{t}} \mid y_{0}\right\}= & \exp \left\{-\mu \mathrm{e}^{-\alpha t} y_{0}-\rho t+\beta\left(\mathrm{e}^{-\alpha t}-1\right) \mu-\frac{\sigma^{2}}{4 \alpha} \mu^{2}\left(\mathrm{e}^{-2 \alpha t}-1\right)\right\} \\
& \times \prod_{i=1}^{m}\left[\frac{\eta_{i} \mathrm{e}^{\alpha t}+\mu}{\eta_{i}+\mu}\right]^{\frac{\rho p_{u} \lambda_{i}}{\alpha}} \times \prod_{j=1}^{n}\left[\frac{\theta_{j} \mathrm{e}^{\alpha t}-\mu}{\theta_{j}-\mu}\right]^{\frac{\rho q_{d} q_{j}}{\alpha}} .
\end{aligned}
$$

Differentiate (4.2) with respect to $\mu$ and put $\mu=0$, then (4.1) is obtained directly.

Proposition 4.2 Assume that the process $y_{t}$ satisfies dynamics (1.2), (1.3), and (1.4). Then the variance of $y_{t}$ is given by

$$
\operatorname{Var}^{Q}\left(y_{t} \mid y_{0}\right)=\left[\frac{\sigma^{2}}{2 \alpha}+\frac{\rho}{\alpha}\left(p_{u} \sum_{i=1}^{m} \frac{\lambda_{i}}{\eta_{i}^{2}}+q_{d} \sum_{j=1}^{n} \frac{q_{j}}{\theta_{j}^{2}}\right)\right]\left(1-\mathrm{e}^{-2 \alpha t}\right) .
$$

Proof We compute the second derivative of (4.2) with respect to $\mu$ and put $\mu=0$, then the second moment of $y_{t}$ is

$$
\begin{aligned}
E^{Q}\left(y_{t}^{2} \mid y_{0}\right)= & \left(\mathrm{e}^{-\alpha t} y_{0}-\beta\left(\mathrm{e}^{-\alpha t}-1\right)\right)^{2}+\frac{\sigma^{2}}{2 \alpha}\left(1-\mathrm{e}^{-2 \alpha t}\right) \\
& -\frac{2 \rho}{\alpha}\left(p_{u} \sum_{i=1}^{m} \frac{\lambda_{i}}{\eta_{i}}+q_{d} \sum_{j=1}^{n} \frac{q_{j}}{\theta_{j}}\right)\left(\mathrm{e}^{-\alpha t} y_{0}-\beta\left(\mathrm{e}^{-\alpha t}-1\right)\right)\left(\mathrm{e}^{-\alpha t}-1\right) \\
& +\frac{\rho^{2}}{\alpha^{2}}\left(p_{u} \sum_{i=1}^{m} \frac{\lambda_{i}}{\eta_{i}}+q_{d} \sum_{j=1}^{n} \frac{q_{j}}{\theta_{j}}\right)\left(\mathrm{e}^{-\alpha t}-1\right)^{2} \\
& +\frac{\rho}{\alpha}\left(p_{u} \sum_{i=1}^{m} \frac{\lambda_{i}}{\eta_{i}^{2}}+q_{d} \sum_{j=1}^{n} \frac{q_{j}}{\theta_{j}^{2}}\right)\left(1-\mathrm{e}^{-2 \alpha t}\right) .
\end{aligned}
$$

Therefore, from (4.1) and (4.4), we can get (4.3). The proof is completed.

Replace $y_{t}$ and $-\alpha$ in (1.2) to $L_{t}$ and $v(v>0)$, respectively, and assume that $L_{0}=0$ and $\beta=0$. Then we have the following model:

$$
\mathrm{d} L_{t}=v L_{t} \mathrm{~d} t+\sigma \mathrm{d} B_{t}+\mathrm{d} J_{t}
$$

where $L_{t}$ denotes the aggregate claim amounts accumulated via a stochastic interest rate up to time $t$. Similar to the previous arguments, we get the expectation and variance of the process $L_{t}$.

Proposition 4.3 Assume that the process $L_{t}$ satisfies dynamics (4.5), (1.3), and (1.4). Then the expectation and variance of the process $L_{t}$ are given by

$$
E^{Q}\left(L_{t}\right)=\frac{\rho}{v}\left(p_{u} \sum_{i=1}^{m} \frac{\lambda_{i}}{\eta_{i}}+q_{d} \sum_{j=1}^{n} \frac{q_{j}}{\theta_{j}}\right)\left(\mathrm{e}^{v t}-1\right)
$$


and

$$
\operatorname{Var}^{Q}\left(L_{t}\right)=\left[\frac{\sigma^{2}}{2 v}+\frac{\rho}{v}\left(p_{u} \sum_{i=1}^{m} \frac{\lambda_{i}}{\eta_{i}^{2}}+q_{d} \sum_{j=1}^{n} \frac{q_{j}}{\theta_{j}^{2}}\right)\right]\left(\mathrm{e}^{2 v t}-1\right) .
$$

Corollary 4.1 Assume that the process $L_{t}$ satisfies dynamics (4.5), (1.3), and (1.4) for $\sigma=0$, $v=\delta, p_{u}=1, q_{d}=0$, and $m=2$. Then the expectation and variance of the process $L_{t}$ are given by

$$
E^{Q}\left(L_{t}\right)=\frac{\rho}{\delta}\left(\frac{\lambda_{1}}{\eta_{1}}+\frac{\lambda_{2}}{\eta_{2}}\right)\left(\mathrm{e}^{\delta t}-1\right)
$$

and

$$
\operatorname{Var}^{Q}\left(L_{t}\right)=\frac{\rho}{\delta}\left(\frac{\lambda_{1}}{\eta_{1}^{2}}+\frac{\lambda_{2}}{\eta_{2}^{2}}\right)\left(\mathrm{e}^{2 \delta t}-1\right) .
$$

Remark 4.1 Léveillé and Garrido [39], Jang [37] studied the moments of the aggregate accumulated claim amounts and obtained the conclusions (4.8) and (4.9). Since (4.9) is a special case of (4.7) for $\sigma=0, p_{u}=1, q_{d}=0$, and $m=2$, we extend the corresponding results in Léveillé and Garrido [39] and Jang [37].

\section{Conclusion}

In this paper, we introduce the concept of Vasicek model with mixed-exponential jumps. We assume that the jump arrival process follows a Poisson process and the jump sizes follow a mixed-exponential distribution. We first describe the structure of the generalized Vasicek model. Then we give the infinitesimal generator of the vector process $\left(Y_{t}, y_{t}, t\right)$, where $y_{t}$ is the generalized Vasicek model and $Y_{t}$ is the integrated process of $y_{t}$. By means of the piecewise deterministic Markov process theory and the martingale property, we derive the explicit forms of the Laplace transforms for the distribution of the generalized Vasicek model and its integrated process. Based on the conclusions obtained, we discuss some applications of the Laplace transforms in finance and insurance. We present the explicit expressions for the price of the default-free zero-coupon bond, the price of the European put option on the zero-coupon bond and the moments of the aggregate accumulated claims. Some numerical calculations are also provided in this paper to illuminate the dynamic relationships between the prices and the parameters used in the model.

As we know, in practice, we might need to employ one of the heavy-tailed distributions for jump size, such as those of Pareto, Gumbel, and Fréchet, in the cases of extreme insurance losses or sudden extreme interest rate rises. However, since it is not likely for us to obtain the explicit expressions for the Laplace transforms of the distributions of $y_{t}$ and $Y_{t}$, we need to use some numerical approaches to calculate the insurance premiums, the zero-coupon bond, and the European put option prices. 


\section{Competing interests}

The authors declare that they have no competing interests.

\section{Authors' contributions}

All authors contributed equally to the writing of this paper. All authors read and approved the final manuscript.

\section{Author details}

'School of Mathematics and Finance, Chuzhou University, Chuzhou, China. ${ }^{2}$ College of Mathematics and Computer Science, Tongling University, Tongling, China. ${ }^{3}$ Institute of Mathematics and Physics, Suzhou University of Science and Techonology, Suzhou, China.

\section{Publisher's Note}

Springer Nature remains neutral with regard to jurisdictional claims in published maps and institutional affiliations.

Received: 3 December 2017 Accepted: 8 April 2018 Published online: 17 April 2018

\section{References}

1. Vasicek, O.: An equilibrium characterization of the term structure. J. Financ. Econ. 5, 177-188 (1977)

2. Cox, J., Ingersoll, J., Ross, S.: A theory of term structure of interest rates. Econometrica 53, 385-407 (1985)

3. Hull, J., White, A.: Pricing interest-rate-derivative securities. Rev. Financ. Stud. 3(4), 573-592 (1990)

4. Patie, P.: On a martingale associated to generalized Ornstein-Uhlenbeck processes and an application to finance. Stoch. Process. Appl. 115(4), 593-607 (2005)

5. Shimko, D., Tejima, N., Van Deventer, D.: The pricing of risky debt when interest rates are stochastic. J. Fixed Income 3(2), 58-65 (1993)

6. Mamon, R.S.: Three ways to solve for bond prices in the Vasicek model. J. Appl. Math. Decis. Sci. 8(1), 1-14 (2004)

7. Liang, L.Z., Lemmens, D., Tempere, J.: Generalized pricing formulas for stochastic volatility jump diffusion models applied to the exponential Vasicek model. Eur. Phys. J. B 75, 335-342 (2010)

8. Siu, T.K.: Bond pricing under a Markovian regime-switching jump-augmented Vasicek model via stochastic flows. Appl. Math. Comput. 216(11), 3184-3190 (2010)

9. Nowman, K.B.: Modelling the UK and Euro yield curves using the generalized Vasicek model: empirical results from panel data for one and two factor models. Int. Rev. Financ. Anal. 19(5), 334-341 (2010)

10. Chen, H.M., Hu, C.F.: A relaxed cutting plane algorithm for solving the Vasicek-type forward interest rate model. Eur. J. Oper. Res. 204(2), 343-354 (2010)

11. Qiu, D., Hu, Y., Wang, L.: The application of option pricing theory in participating life insurance pricing based on Vasicek model. In: Wu, D. (ed.) Quantitative Financial Risk Management. Computational Risk Management, vol. 1, pp. 39-46. Springer, Berlin (2011). https://doi.org/10.1007/978-3-642-19339-2_4

12. Liang, J., Ma, J.M., Wang, T., Ji, Q.: Valuation of portfolio credit derivatives with default intensities using the Vasicek model. Asia-Pac. Financ. Mark. 18(1), 33-54 (2011)

13. Su, X.N., Wang, W.S.: Pricing options with credit risk in a reduced form model. J. Korean Stat. Soc. 41, 437-444 (2012)

14. Liang, X., Wang, G.J., Dong, Y.H.: A Markov regime switching jump-diffusion model for the pricing of portfolio credit derivatives. Stat. Probab. Lett. 83, 373-381 (2013)

15. Dong, Y.H.: Pricing dynamic guaranteed funds with stochastic barrier under Vasicek interest rate model. Chinese J. Appl. Probab. Statist. 29(3), 237-245 (2013)

16. Ishitani, K.: A fast wavelet expansion technique for evaluation of portfolio credit risk under the Vasicek multi-factor model. Jpn. J. Ind. Appl. Math. 31(1), 1-24 (2014)

17. Xiao, W.L., Zhang, W.G., Zhang, X.L., Chen, X.Y.: The valuation of equity warrants under the fractional Vasicek process of the short-term interest rate. Phys. A, Stat. Mech. Appl. 394(15), 320-337 (2014)

18. Branger, N., Kraft, H., Meinerding, C.: Partial information about contagion risk, self-exciting processes and portfolio optimization. J. Econ. Dyn. Control 39, 18-36 (2014)

19. Nowak, P., Romaniuk, M.: Catastrophe bond pricing for the two-factor Vasicek interest rate model with automatized fuzzy decision making. Soft Comput. 21(10), 2575-2597 (2017)

20. Chang, H., Chang, K.: Optimal consumption-investment strategy under the Vasicek model: HARA utility and Legendre transform. Insur. Math. Econ. 72, 215-227 (2017)

21. Hajipour, M., Malek, A.: High accurate modified WENO method for the solution of Black-Scholes equation. Comput. Appl. Math. 34(1), 125-140 (2015)

22. Hajipour, M., Malek, A.: Efficient high-order numerical methods for pricing of options. Comput. Econ. 45(1), 31-47 (2015)

23. Jajarmi, A., Hajipour, M., Baleanu, D.: New aspects of the adaptive synchronization and hyperchaos suppression of a financial model. Chaos Solitons Fractals 99, 285-296 (2017)

24. Beliaeva, N., Nawalkha, S.K., Soto, G.M.: Pricing American interest rate options under the jump-extended Vasicek model. J. Deriv. 16(1), 29-43 (2008). https://doi.org/10.2139/ssrn.966282

25. Backus, D., Foresi, S., Wu, L.R.: Macro-economic foundations of higher-order moments in bond yields. Working paper, New York University (1997)

26. Das, S.R., Foresi, S.: Exact solutions for bond and option prices with systematic jump risk. Rev. Deriv. Res. 1(1), 7-24 (1996)

27. Das, S.R.: The surprise element: jumps in interest rates. J. Econom. 106(1), 27-65 (2002)

28. Johannes, M.: The statistical and economic role of jumps in continuous-time interest rate models. J. Finance 59 , 227-260 (2004)

29. Piazzesi, M.: Bond yields and the federal reserve. J. Polit. Econ. 113(2), 311-344 (2005)

30. Chacko, G., Das, S.R.: Pricing interest rate derivatives: a general approach. Rev. Financ. Stud. 15(1), 195-241 (2002) 
31. Su, X.N., Wang, W.S.: Pricing options with credit risk in a reduced form model. J. Korean Stat. Soc. 41(4), 437-444 (2012)

32. Cai, N., Kou, S.G.: Option pricing under a mixed-exponential jump diffusion model. Manag. Sci. 57, 2067-2081 (2011)

33. Botta, R.F., Harris, C.M.: Approximation with generalized hyperexponential distributions: weak convergence results. Queueing Syst. 1(2), 169-190 (1986)

34. Davis, M.H.A.: Piecewise deterministic Markov processes: a general class of non-diffusion stochastic models. J. R. Stat. Soc., Ser. B 46, 353-388 (1984)

35. Rolski, T., Schmidli, H., Schmidt, V., Teugels, J.L.: Stochastic Processes for Insurance and Finance. Wiley Series in Probability and Statistics. Wiley, New York (1998). https://doi.org/10.1002/9780470317044

36. Jacobsen, M.: Point Process Theory and Applications. Marked Point and Piecewise Deterministic Processes. Birkhäuser, Boston (2006)

37. Jang, J.: Martingale approach for moments of discounted aggregate claims. J. Risk Insur. 71(2), 201-211 (2004)

38. Duffie, D., Pan, J., Singleton, K.: Transform analysis and asset pricing for affine jump-diffusions. Econometrica 68 1343-1376 (2000)

39. Léveillé, G., Garrido, J.: Moments of compound renewal sums with discounted claims. Insur. Math. Econ. 28, 217-231 (2001)

\section{Submit your manuscript to a SpringerOpen ${ }^{\circ}$ journal and benefit from:}

- Convenient online submission

- Rigorous peer review

- Open access: articles freely available online

- High visibility within the field

- Retaining the copyright to your article

Submit your next manuscript at $\boldsymbol{~ s p r i n g e r o p e n . c o m ~}$ 